\title{
A Fellowship of RING1 Maintains AML Stem Cells
}

\section{David M. Heery}

University of Nottingham

Polycomb-related complexes (PRC1 and PRC2) are transcriptional repressors that function as key regulators of the self-renewal and differentiation (cell lineage specification) pathways in stem cells. In this issue of Blood, Shima et al [1] report that two ubiquitin ligases (RING1A and RING1B) which are key components of PRC1 complexes, are essential for the establishment and maintenance of acute myeloid leukemias (AMLs) in mouse models. AMLs frequently harbour non-random, recurrent chromosomal translocations involving fusions of genes encoding transcription factors, chromatin (epigenetic) regulators or signalling proteins. The resultant oncogenic fusion proteins such as MLL-AF10, AML1-ETO or MOZ-TIF2, can subvert normal self-renewal and differentiation pathways and promote the proliferation of leukemia initiating cells and their progeny. Revealing the mode of action of leukemic fusion proteins and the processes that underpin them is important to advance new strategies to treat AML.

The Shima et al [1] study focussed on MOZ-TIF2, which results from translocations involving the KAT6A and NCOA2 genes, encoding the histone acetyltransferase $\mathrm{MOZ}$ and the Nuclear Receptor Coactivator TIF2, respectively. This resultant expressed protein contains the Nterminal half of $\mathrm{MOZ}$ comprising a histone acetyltransferase domain and several histone recognition domains fused to the C-terminal portion of TIF2, a region that contains domains for binding other epigenetic regulators such as the CBP/p300 acetyltransferases. Expression of MOZ-TIF2 protein in bone marrow progenitors is sufficient to induce leukemia in mice, revealing that the oncogenic fusion protein has a causal role in leukemogenesis [2]. However, the modes of action of the MOZ-TIF2 and other leukemic fusion proteins (e.g. 
MLL-AF10 and AML1-ETO) are complex, as they can promote changes in histone modifications, impact on the stability or recruitment of other epigenetic regulators to chromatin, and thus produce substantial changes in gene expression. For example, MOZTIF2 interacts with haematopoietic transcription factors including AML1/Runx1 and Pu.1 [3], as well as chromatin regulators such as $\mathrm{CBP} / \mathrm{p} 300, \mathrm{KDM} 4 \mathrm{C}, \mathrm{PRMT1}$ and the ING/BRPF1/EAF6 complex [4-6]. This complexity of function potentially exacerbates the challenge of developing bespoke therapies for AMLs driven by different translocation events.

The study by Shima et al [1] combined with several other reports [7, 8] now reveals the importance of the RING1A and RING1B ubiquitin ligases in facilitating the maintenance and survival of leukemia initiating cells derived from several different oncogenic fusions. RING1A and RING1B are core components of PRC1 polycomb complexes which repress genes that promote differentiation. A major function of RING1 (the collective term for the RING1A and RING1B proteins) is to add a ubiquitin polypeptide to lysine residue K119 of the histone $\mathrm{H} 2 \mathrm{~A}$. This event can be facilitated by PRC2-mediated deposition of $\mathrm{H} 3 \mathrm{~K} 27$ me3, a repressive mark that can be recognised by other components of PRC1. The tri-methylation of H3K27, together with H2AK119-Ub is believed to tether RNA Polymerase II complexes on regions that also contain active marks such as $\mathrm{H} 3 \mathrm{~K} 4 \mathrm{me} 3$, and therefore such 'bivalent' promoters may be poised for expression in response to differentiation signals. Shima et al [1] showed that conditional inactivation of RING1 results in differentiation of the MOZ-TIF2 induced leukemic cells into mature myeloid cells. As a consequence, the loss of H2AK119-Ubi releases the brake on expression of a cohort of differentiation promoting genes that includes the cell cycle regulator $C D K N 2 A$.

$C D K N 2 A$ is a tumour suppressor gene that encodes the INK4A/ARF proteins (also termed p16 and p19), and many studies have shown that repression of this gene by Polycomb complexes and other regulators is vital to maintain the self-renewal capacity of stem cells. Interestingly, both wild type MOZ and MOZ-TIF2 play a role in the repression of CDKN2A to inhibit senescence in normal and leukemic stem cells, respectively $[9,10]$, 
although the mechanism is not fully elucidated. Consistent with other studies [7], inactivation of RING1A/B resulted in derepression of $C D K N 2 A$, accompanied by concomitant loss of PRC1-mediated H2A ubiquitylation and H3K27me3, which is catalysed by PRC2. However, using combined knockout experiments, these studies revealed that inactivation of $C D K N 2 A$ gene by genetic ablation was not sufficient by itself to restore fusion protein-dependent leukemogenicity [1,7], suggesting other targets are important in this process.

In attempts to identify other players, Shima et al [1] turned to transcriptomic profiling to identify genes that are derepressed by RING1 inactivation and identified a strong upregulation of GLIS2, which encodes a Krüppel-like zinc finger protein. GLIS2 is involved in reciprocal translocations with CBFA2T3/ETO2 gene in pediatric acute megakaryoblastic leukemia patients, and Shima et al [1] provide further evidence for a role for GLIS2 in the differentiation of myeloid progenitors. However, enforced overexpression of GLIS2 in MOZTIF2 transformed cells only delayed rather than blocked AML progression, and similarly, simultaneous inactivation of GLIS2 and RING1 did not rescue MOZ-TIF2 induced leukemogenesis. However, the inactivation of GLIS2 in addition to RING1 preserved a population of AML-derived granulocyte-macrophage progenitors (L-GMPs). These results support a role for GLIS2 in myeloid differentiation, but suggest that GLIS2 and CDKN2A are not the only RING1 targets whose suppression facilitates leukemogenesis.

In summary, PRC1 Polycomb complexes appear to be key guardians of normal haematopoietic stem cell populations, in addition to being vital to the propagation of AMLs arising from different recurrent chromosomal translocations. The advances reviewed here show that RING1 ubiquitin ligase activity is essential to these processes [1, 7], perhaps identifying a potential Achilles' heel to target in AML initiating cells. 
1. Shima, H., et al., Ring1A and Ring $1 B$ inhibit expression of Glis2 to maintain murine MOZ-TIF2 AML stem cells. Blood, 2018.

2. Deguchi, K., et al., MOZ-TIF2-induced acute myeloid leukemia requires the MOZ nucleosome binding motif and TIF2-mediated recruitment of CBP. Cancer Cell, 2003. 3(3): p. 259-71.

3. Aikawa, Y., et al., PU.1-mediated upregulation of CSF1R is crucial for leukemia stem cell potential induced by MOZ-TIF2. Nat Med, 2010. 16(5): p. 580-5, 1p following 585.

4. Kindle, K.B., et al., MOZ-TIF2 inhibits transcription by nuclear receptors and $p 53$ by impairment of CBP function. Mol Cell Biol, 2005. 25(3): p. 988-1002.

5. Ullah, M., et al., Molecular architecture of quartet MOZ/MORF histone acety/transferase complexes. Mol Cell Biol, 2008. 28(22): p. 6828-43.

6. Cheung, N., et al., Targeting Aberrant Epigenetic Networks Mediated by PRMT1 and KDM4C in Acute Myeloid Leukemia. Cancer Cell, 2016. 29(1): p. 32-48.

7. Rossi, A., et al., Maintenance of leukemic cell identity by the activity of the Polycomb complex PRC1 in mice. Sci Adv, 2016. 2(10): p. e1600972.

8. van den Boom, V., et al., Non-canonical PRC1.1 Targets Active Genes Independent of H3K27me3 and Is Essential for Leukemogenesis. Cell Rep, 2016. 14(2): p. 332-46.

9. Sheikh, B.N., et al., MOZ (MYST3, KAT6A) inhibits senescence via the INK4A-ARF pathway. Oncogene, 2015. 34(47): p. 5807-20.

10. Largeot, A., et al., Expression of the MOZ-TIF2 oncoprotein in mice represses senescence. Exp Hematol, 2016. 44(4): p. 231-7 e4.

Figure 1

Schematic representation of the action of RING1A and RING1B proteins in promoting the maintenance of AML cells derived from MOZ-TIF2, MLL-AF10, PML-RAR or AML1-ETO fusion proteins. RING1 proteins associated with polycomb PRC1 complexes catalyse the ubiquitylation of lysine $\mathrm{K} 119$ of histone $\mathrm{H} 2 \mathrm{~A}$ (H2AK119-Ubi). This modification, in addition to the H3K27me3, contributes to repression of genes including GLIS2 and CDKN2A, promoting the self-renewal of leukemia-initiating cells. As these normally 'bivalent' promoters also contain the activating chromatin modification $\mathrm{H} 3 \mathrm{~K} 4 \mathrm{me} 3$, inactivation of RING1 results in derepression of GLIS2, CDKN2A and other genes, promoting differentiation. 
